\title{
Carbon fiber skeleton/silver nanowires composites with tunable negative permittivity behavior
}

\author{
Yan $\mathrm{An}^{1}$, Jinyuan Qin ${ }^{1}$, Kai Sun ${ }^{1, *}$, Jiahong Tian $^{1}$, Zhongyang Wang ${ }^{2,}$, Yaman Zhao ${ }^{1}$, \\ Xiaofeng $\mathrm{Li}^{1}$, and Runhua Fan $^{1}$ \\ ${ }^{1}$ College of Ocean Science and Engineering, Shanghai Maritime University, Shanghai 201306, PR China \\ 2 State Key Lab of Metal Matrix Composites, School of Materials Science and Engineering, Shanghai Jiao Tong University, \\ Shanghai 200240, PR China
}

Received: 13 November 2020 / Accepted: 25 December 2020

\begin{abstract}
With the development of periodic metamaterials, more attention has been paid to negative permittivity behavior due to great potential applications. In this paper, silver nanowires (AgNWs) were introduced to the porous carbon fibers (CFS) by an impregnation process to prepare CFS/AgNWs composites with different content of AgNWs and the dielectric property was investigated. With the formation of conductive network, the Drude-like negative permittivity was observed in CFS/AgNWs composites. With the increase of $\mathrm{AgNWs}$, the connectivity of conductive network became enhanced, the conductivity gradually increases, and the absolute value of the negative dielectric constant also increases to $8.9 \times 10^{4}$, which was ascribed to the enhancement of electron density of the composite material. Further investigation revealed that the inductive characteristic was responsible for the negative permittivity.
\end{abstract}

Keywords: Negative permittivity / metacomposites / plasma oscillation / inductive characteristic

\section{Introduction}

In the past decades, electromagnetic metamaterials with negative permittivity and negative permeability have attracted a wide range of attention due to their unique electromagnetic properties, such as negative refraction [1], perfect imaging [2] and super absorption [3,4], etc. The application of electromagnetic metamaterials in the antenna system can improve the directivity and tunability and reduce the size of the antenna $[5,6]$. With the development of science and technology, traditional stealth technology and wave-absorbing materials have been unable to meet the requirements of application. In 2006 , Pendry et al. [7] first proposed the idea of using metamaterials to prepare an invisibility cloak, and gave the parameters required for the design of the cloak, which theoretically proved the feasibility of the idea. Then, a twodimensional invisibility cloak or carpet is prepared and applied to the microwave frequency band. The feasibility of electromagnetic stealth is verified experimentally $[8,9]$. In addition, the high energy density and low loss and breakdown strength make the composite material ideal material for high performance dielectric energy storage

\footnotetext{
* e-mail: kais@shmtu.edu.cn
}

capacitors [10,11]. The electromagnetic metamaterials have a great value in the fields of wireless communication [12], electromagnetic absorption [13] and shielding [14], etc. To date, electromagnetic metamaterials are mainly classified into two types, namely the periodic metamaterials and random metamaterials. In general, periodic metamaterials are composed of structural units in the scale of subwavelength and their electromagnetic performance mainly depends on geometric shape, size and arrangement of the structure units, rather than on their chemical composition and microstructure. As a supplementary of periodic metamaterials, random metamaterials termed as metacomposites are favored owing to their facile fabrication, low cost and tunable properties based on intrinsic properties of materials, such as composition and miscrostructure [15-17].

For the random metacomposites, the negative permittivity can be obtained by appropriately tailoring their microstructures. It indicates that the negative permittivity is caused by the plasma oscillation of delocalized electrons [18]. In fact, the negative permittivity was initially observed in metal materials below the oscillation frequency. For the pure metal bulk, the oscillation frequency is usually observed at visible or ultraviolet frequency region, so the absolute values of negative permittivity can be very huge in radio frequency region $[19,20]$. There is an urgent 

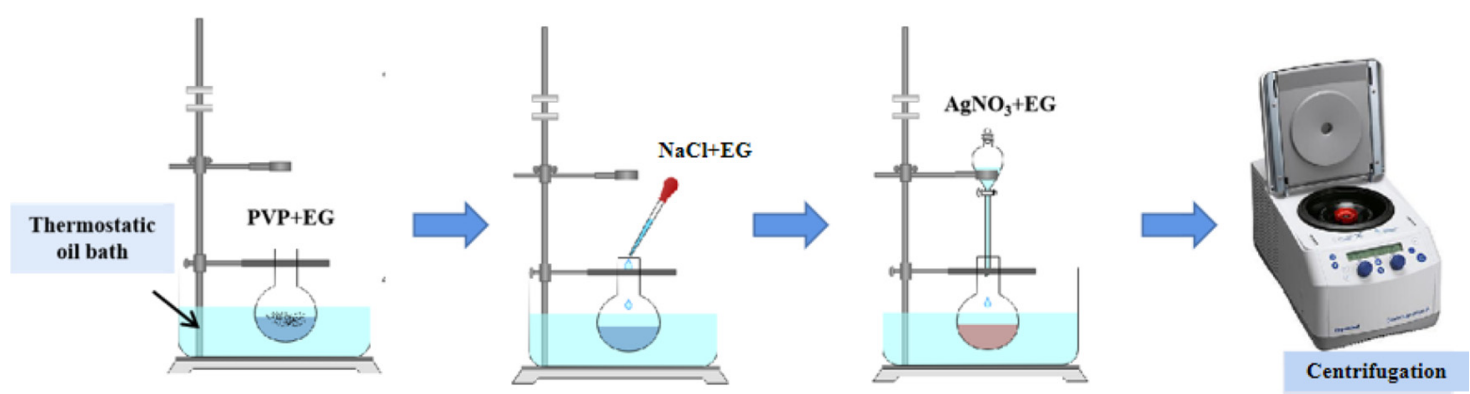

Fig. 1. The preparation schematic diagram of AgNWs.

requirement in obtaining tunable negative permittivity with a low-frequency plasmon state to meet the practical applications. In our previous investigation, metal particles such as silver and nickel were diluted in ceramic matrix to decrease the plasma oscillation frequency to radio frequency $[21,22]$. Similarly, Tsutaoka et al. [23-26] observed negative permittivity with a low frequency plasmon state in resin-based composites with copper or nickel particles. Moreover, Park et al. [27] introduced chain-like nickel to polyvinylidene fluoride and achieve the tunable negative permittivity. Compared with that of metal materials, there are fewer investigations focused on carbon materials, and the mechanism of negative permittivity should be further revealed.

It indicates that the negative permittivity was in close association with the connected network. In theory, introducing conductive fillers can contribute to the form of connected network, leading to a strongly negative permittivity. Based on this idea, herein the porous carbon fiber skeleton (CFS) was selected as the matrix to load onedimensional silver nanowires (AgNWs) by an impregnation method. After introducing AgNWs, the absolute values of negative permittivity significantly increased, which was attributed to the enhanced connectivity of the conductive network. The tunable negative permittivity behavior was observed in CFS/AgNWs composites by adjusting the composition and microstructure. In addition, the relationship between the impedance and permittivity was studied. It demonstrated that the negative permittivity was ascribed to inductive character of conductive network.

\section{Experimental procedure}

\subsection{Materials}

The carbon fiber skeleton is provided by Hangzhou Vulcan New Materials Technology Co., Ltd (China). In addition, silver nitrate $\left(\mathrm{AgNO}_{3}\right.$, purity $\left.99.99 \%\right)$, polyvinylpyrrolidone (PVP) with an average molecular weight $M_{w}$ of 30000 , sodium chloride $(\mathrm{NaCl})$, ethylene glycol (EG) and anhydrous ethanol were purchased from Sinopharm Chemical Reagent Co., Ltd (China). All the raw materials were used without any treatment.

\subsection{Preparation of AgNWs}

Figure 1 shows the preparation schematic diagram of AgNWs. AgNWs were synthesized by a polyol method.
Firstly, add $5.8 \mathrm{~g}$ of PVP powder and $184 \mathrm{ml}$ of EG into the flask. Then put the flake in oil bath pot to uniformly heat from room temperature (RT) to $195^{\circ} \mathrm{C}$ along with magnetic stirring. Subsequently, add $0.036 \mathrm{~g}$ of $\mathrm{NaCl}$ into $60 \mathrm{ml}$ of EG to completely dissolve, and then slowly drop $6 \mathrm{ml}$ of the prepared $\mathrm{NaCl}$ solution into the flask. After that, dissolve $1.529 \mathrm{~g}$ of $\mathrm{AgNO}_{3}$ into $10 \mathrm{ml}$ of $\mathrm{EG}$ and drop the $\mathrm{AgNO}_{3}$ solution into flask. After reacting for $15 \mathrm{~min}$, the solution naturally cooled to RT, then centrifuged at $10000 \mathrm{rpm}$ to collect the AgNWs. Finally, the collected AgNWs were repeatedly washed with deionized water to remove PVP and EG until the supernatant became colorless. In this experiment, $\mathrm{AgNO}_{3}$ was used as the source of AgNWs and PVP was used as the structureoriented reagent. $\mathrm{EG}$ and $\mathrm{NaCl}$ were used as reducing agent and additive, respectively.

\subsection{Preparation of CFS/AgNWs composites}

The CFS was used as matrix. Before the impregnation process, the CFS was completely dried in a vacuum drying oven then obtained its initial mass. The prepared AgNWs were dispersed in deionized water and stirred continuously to form AgNWs suspension. Subsequently, the dried CFS was impregnated into AgNWs suspension. After impregnation process, the CFS was placed in a blast air oven to dry at $120^{\circ} \mathrm{C}$ for $1 \mathrm{~h}$. Finally, CFS/AgNWs composites with different content of AgNWs (0, 0.67, 1.37, 2.08, 3.03, 3.68 and $4.03 \mathrm{wt} \%$ ) were prepared by repeatedly impregnation and drying.

\subsection{Characterization and measurement}

The microstructure and distribution of the samples were observed by field emission scanning electron microscope (FESEM: JSM 7500F, Japan). X-ray diffraction (XRD: PANalytical X'pert PRO, the Netherlands) was used to analyze the phase and crystal structure of the prepared materials. The scanning range was $10-90^{\circ}$, and the scanning speed was $5^{\circ} \mathrm{min}$. An LCR meter (Keysight, E4980AL, USA) was used to test the dielectric properties of the samples in the frequency range of $10 \mathrm{kHz}-1 \mathrm{MHz}$. After the equipment was calibrated and compensated, the sample was placed between two electrodes for measurement. The impedance and capacitance of the samples were measured directly by LCR meter at room temperature. The permittivity $\left(\varepsilon^{\prime}\right)$, impedance modulus $(|Z|)$ and $\mathrm{AC}$ 


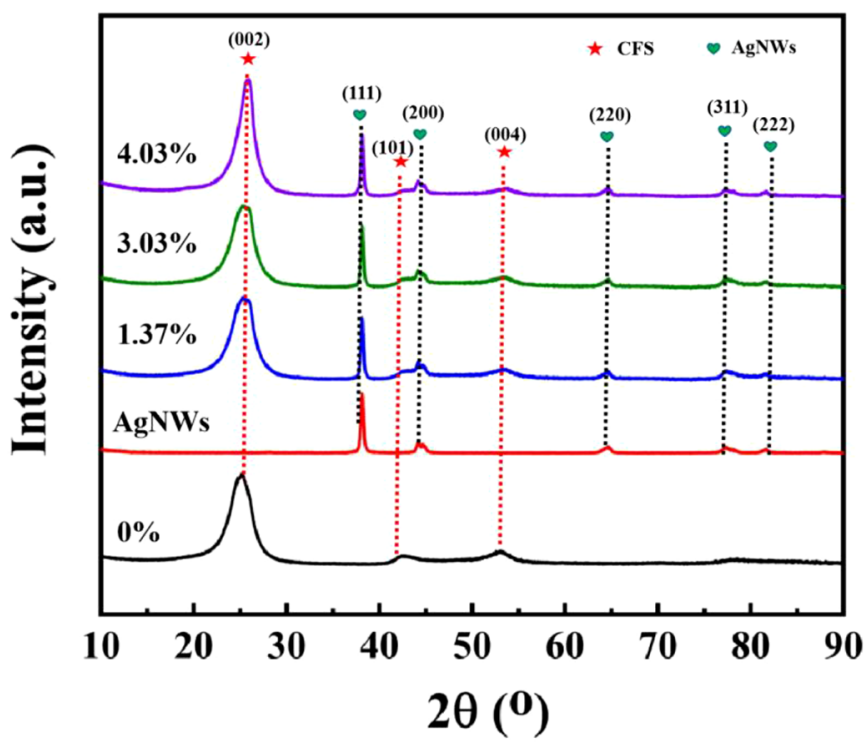

Fig. 2. XRD patterns of CFS and CFS/AgNWs composites with different content of AgNWs.

conductivity $\left(\sigma_{\mathrm{ac}}\right)$ are calculated as follows,

$$
\begin{gathered}
\varepsilon^{\prime}=\frac{C d}{\varepsilon_{0} A} \\
|Z|=\sqrt{\left|Z^{\prime}\right|^{2}+\left|Z^{\prime \prime}\right|^{2}} \\
\sigma_{\mathrm{ac}}=\frac{d}{R S}
\end{gathered}
$$

where $d$ is the sample thickness, $A$ is the area of electrode, $C$ is capacitance $\varepsilon_{0}$ is the vacuum dielectric constant $\left(8.85 \times 10^{-12} \mathrm{~F} / \mathrm{m}\right), Z^{\prime}$ and $Z^{\prime \prime}$ are the real and imaginary impedance, $R$ is the resistance and $S$ is the area of sample.

\section{Results and discussion}

The XRD phase analysis of CFS/AgNWs composites is shown in Figure 2. The XRD test conditions are scanning speed $5^{\circ} / \mathrm{min}$ and scanning range $10-90^{\circ}$ at room temperature. For the pure CFS, three distinct characteristic peaks were observed at about $26^{\circ}, 43^{\circ}$ and $53^{\circ}$, corresponding to (002), (101) and (004) crystal surfaces, respectively. In the radial direction of carbon fiber, the (002) crystal surface is closely related to the interlaminar space. The presence of strong diffraction peaks at about $26^{\circ}$ corresponding to (101) and (004) crystal faces indicated that the CFS had a high graphitization [28], which was conducive to the increase of conductivity. When AgNWs were introduced into the CFS, five peaks were observed at around $38.1^{\circ}, 43.8^{\circ}, 64.1^{\circ}, 77.3^{\circ}$ and $81.8^{\circ}$, consistent with JCPDS card (\#00-004-0783) of silver [29]. With the increase of AgNWs, the diffraction intensity of silver
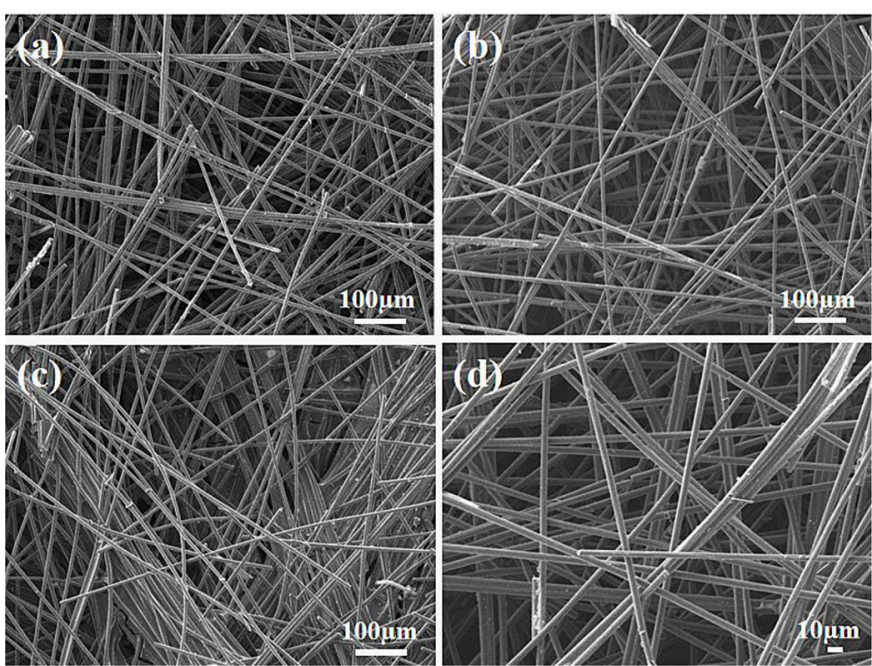

Fig. 3. The SEM images of CFS/AgNWs composites with different content of AgNWs. (a-d) represents 0, 1.37, 3.03 and $4.03 \mathrm{wt} \%$ content of AgNWs.

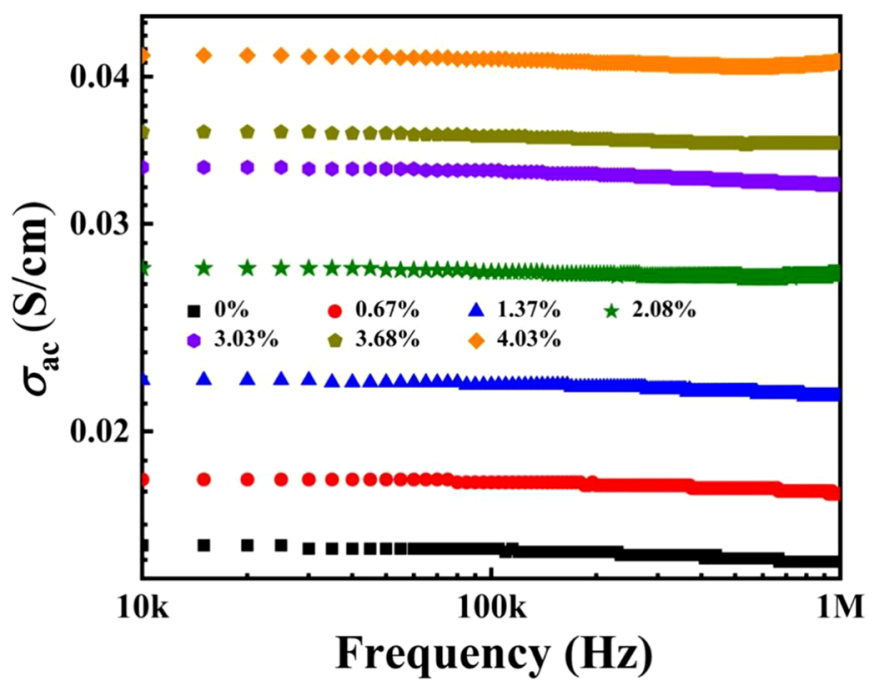

Fig. 4. The AC conductivity spectra of CFS / AgNWs composites with different content of AgNWs.

increased, but the position of characteristic peak did not shift. According to XRD analysis, it indicated that CFS/ AgNWs composites were prepared.

Figure 3 shows the SEM images of the CFS/AgNWs composites with different AgNWs content. The AgNWs in the composites were uniformly distributed on the CFS. When the content of AgNWs was $0.67 \mathrm{wt} \%$, AgNWs distributed in the CFS in isolation. With the increase of AgNWs content, AgNWs connected with each other and a new network formed among the CFS, so that the connectivity of the conductive network became enhanced.

Figure 4 shows the AC conductivity spectra of CFS/ AgNWs composites with different content of AgNWs. Pure CFS had a relatively low conductivity in the test frequency 


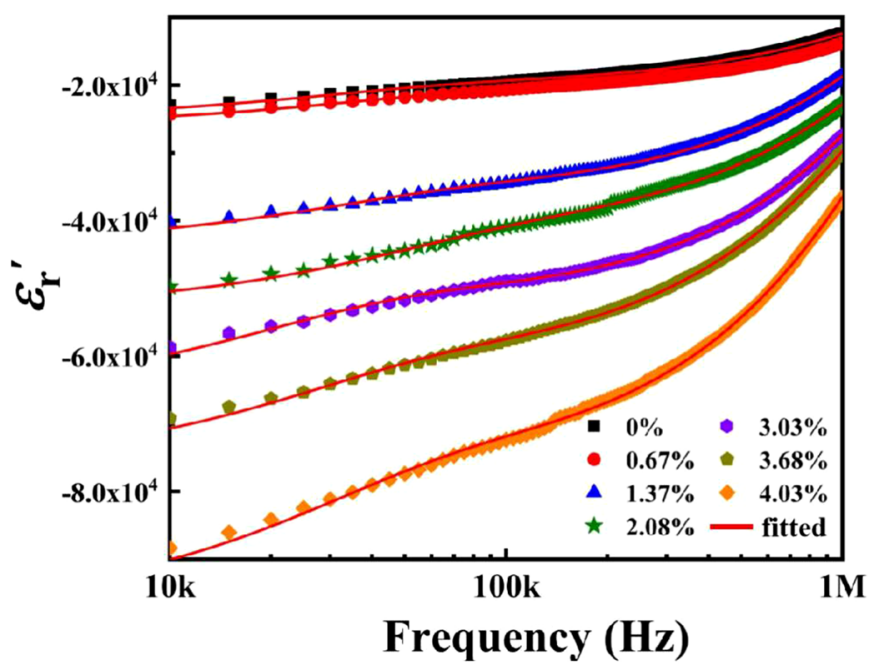

Fig. 5. The permittivity spectra of CFS/ AgNWs composites with different content of AgNWs (0-4.03 wt\%). The solid lines present the fitted results based on the Drude model.

range. When AgNWs were introduced to the CFS, the conductivity of the composites was effectively improved. With the increase of AgNWs, the electrical conductivity of the resulting composites got enhanced. On the other hand, the conductivity gradually decreased in high frequency region, which should be ascribed to the skin effect. As is well known, the pure CFS possessed a good conductivity after graphitization, so CFS can be regarded as a conductor. The connectivity of the conductive network became enhanced due to the existence of AgNWs. Hence, CFS/AgNWs composites exhibited metal-like characteristic. Therefore, the conductivity decreased with the increase of frequency owing to skin effect.

The dielectric properties of CFS/PVA composites were discussed as above. The PVA of insulation property was introduced into the CFS skeleton to realize the regulation of weak negative dielectric properties by changing the electron concentration [30]. In this paper, the innovation is reflected in the introduction of AgNWs with strong conductivity on the basis of CFS, which makes it easier to form a conductive network with increased electron concentration, so as to study the regulation of dielectric properties. Figure 5 shows the permittivity spectra of CFS/ AgNWs composites with different content of AgNWs. For the pure CFS, the permittivity was negative in the whole test frequency. With the increase of $\mathrm{AgNWs}$, the negative permittivity behavior was also maintained in CFS/AgNWs composites. Meanwhile, the absolute values of negative permittivity increased for the composites with more content of AgNWs. On the other hand, along with the increase of AgNWs, the frequency dispersive behavior became stronger, especially in the composites with $4.03 \mathrm{wt} \%$ AgNWs. The pure CFS possessed a high conductivity after graphitization. After introducing AgNWs to the CFS, the conductive AgNWs contributed to the connectivity of network, leading to the increase of electron concentration. Drude model was used to explore the mechanism of negative permittivity, which can be expressed as follows [31-33],

$$
\begin{gathered}
\varepsilon_{r}^{\prime} \omega=1-\frac{\omega_{p}^{2}}{\omega^{2}+\omega_{\tau}^{2}} \\
\omega_{p}=\sqrt{\frac{n_{\mathrm{eff}} e^{2}}{m_{\text {eff } \varepsilon_{0}}}}
\end{gathered}
$$

where $\omega_{\mathrm{p}}\left(\omega_{\mathrm{p}}=2 \pi f_{\mathrm{p}}\right)$ is the plasma angular frequency, $f_{\mathrm{p}}$ is the plasma frequency, $\omega$ is the angular frequency of the applied electric field, $\omega_{\tau}\left(\omega_{\tau}=2 \pi f_{\tau}\right)$ is the damping angular frequency, $\varepsilon_{0}$ is permittivity of vacuum $\left(8.85 \times 10^{-12} \mathrm{~F} / \mathrm{m}\right)$, $n_{\text {eff }}$ is the effective concentration of conduction electrons, $m_{\text {eff }}$ is the effective mass of electron and $e$ is electron charge $\left(1.6 \times 10^{-19} \mathrm{C}\right)$. The solid lines were well fitted by the Drude model.

Equivalent circuit analysis is a simple and effective method to investigate the electrical properties of materials. In order to further reveal the mechanism of negative permittivity, the impedance spectra of CFS/AgNWs composites were analyzed according to equivalent circuit model (Fig. 6). With the increase of AgNWs, the impedance module values gradually decreased, indicating that the conductivity increased. According to the fitted results, for CFS / AgNWs composites with a low content of AgNWs (0-2.08 wt\%), it can be equivalent to the circuit model composed of parallel resistor $\mathrm{R}_{\mathrm{p}}$, capacitor $\mathrm{C}_{1}$, resistor $R_{1}$ and inductor $L_{1}$. In general, the inductor and capacitor show different responses to the $\mathrm{AC}$ electric field, which can be described as inductive reactance $\left(Z_{\mathrm{L}}\right)$ and capacitive reactance $\left(Z_{\mathrm{C}}\right)$. The total reactance is expressed as $Z^{\prime \prime}=Z_{\mathrm{L}}-Z_{\mathrm{C}}$ [21]. On the other hand, for the resulting composites with higher content of AgNWs (3.03-4.03 wt\%), it can be equivalent to the circuit of capacitance $\mathrm{C}$ and resistance $\mathrm{R}_{\mathrm{p}}, \mathrm{R}_{1}, \mathrm{R}_{2}$ and inductance $\mathrm{L}_{1}$ and $\mathrm{L}_{2}$. Due to the enhanced connectivity of conductive network among the composites, the inductive characteristic increased. Further investigation revealed that permittivity and impedance conformed to the following relationship,

$$
\varepsilon_{r}^{\prime}=-\frac{Z^{\prime \prime}}{2 \pi f C_{0}\left(Z^{\prime 2}+Z^{\prime 2}\right)}
$$

where $\mathrm{Z}^{\prime}, \mathrm{Z}^{\prime \prime}$ are the resistance and reactance, $C_{0}$ is the vacuum capacitance and $f$ is test frequency. When the permittivity was negative value, the corresponding reactance was positive value, suggesting an inductive characteristic. Based on this equation, we can draw a brief conclusion that the inductive characteristic is responsible for the negative permittivity.

\section{Conclusions}

In this paper, CFS/AgNWs composites with different content of AgNWs were prepared by an impregnation process. The carbon fiber skeleton shows Drude-like negative permittivity due to itself conductive network. 


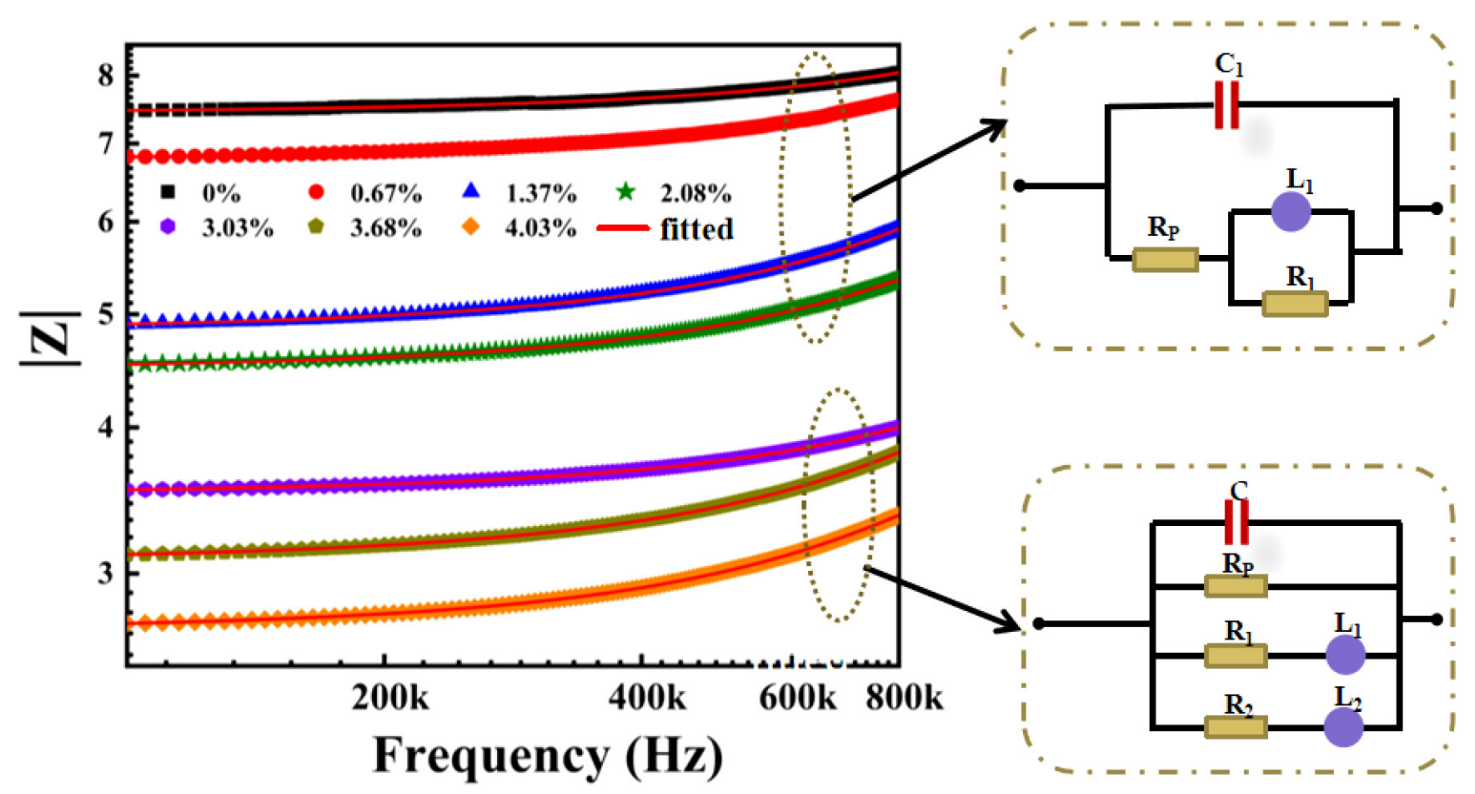

Fig. 6. Impedance module spectra of CFS/AgNWs composites with different content of AgNWs.

When AgNWs were introduced, the absolute values of $\mathrm{CFS} / \mathrm{AgNWs}$ composites increased, which was attributed to the enhanced connectivity of the conductive network, leading to the increase of electron density. In addition, further investigation revealed that the inductive characteristic was responsible for the negative permittivity.

This work was financially supported by the National Natural Science Foundation of China (Grant No. 51803119, 51871146 and 51771108). "Chenguang Program" supported by Shanghai Education Development Foundation and Shanghai Municipal Education Commission (Grant No. 18CG56), the Innovation Program of Shanghai Municipal Education Commission (Grant No. 2019-01-07-00-10-E00053) and Shanghai Engineering Technology Research Centre of Deep Offshore Material (19DZ2253100).

\section{References}

1. D.R. Smith, J.B. Pendry, M.C. Wiltshire, Metamaterials and negative refractive index, Science 305, 788 (2004)

2. M.J. Rust, M. Bates, X. Zhuang, Sub-diffraction-limit imaging by stochastic optical reconstruction microscopy (STORM), Nat. Methods 3, 793 (2006)

3. C.M. Watts, X. Liu, W.J. Padilla, Metamaterial electromagnetic wave absorbers, Adv. Mater. 24, OP98 (2012)

4. M.K. Hedayati, F. Faupel, M. Elbahri, Review of plasmonic nanocomposite metamaterial absorber, Materials 7, 1221 (2014)

5. C. Caloz, T. Itoh, A. Rennings, CRLH metamaterial leakywave and resonant antennas, IEEE Trans. Antennas Propag. 50, 25 (2008)

6. R.W. Ziolkowski, P. Jin, C.-C. Lin, Metamaterial-inspired engineering of antennas, Proc IEEE 99, 1720 (2010)

7. J. Pendry, D. Schurig, D. Smith, Controlling electromagnetic fields, Science 312, 1780 (2006)
8. D. Schurig, J.J. Mock, B.J. Justice, S.A. Cummer, J.B. Pendry, A.F. Starr, D.R. Smith, Metamaterial electromagnetic cloak at microwave frequencies, Science 314, 977 (2006)

9. R. Liu, C. Ji, J.J. Mock, J.Y. Chin, T.J. Cui, D.R. Smith, Broadband ground-plane cloak, Science 323, 366 (2009)

10. L. Sun, Z. Shi, L. Liang, S. Wei, H. Wang, D. Dastan, K. Sun, R. Fan, Layer-structured $\mathrm{BaTiO}_{3} / \mathrm{P}(\mathrm{VDF}-\mathrm{HFP})$ composites with concurrently improved dielectric permittivity and breakdown strength toward capacitive energy-storage applications, J. Mater. Chem. C 8, 10257 (2020)

11. L. Sun, Z. Shi, H. Wang, K. Zhang, D. Dastan, K. Sun, R. Fan, Ultrahigh discharge efficiency and improved energy density in rationally designed bilayer polyetherimide$\mathrm{BaTiO}_{3} / \mathrm{P}(\mathrm{VDF}-\mathrm{HFP})$ composites, J. Mater. Chem. A 8, 5750 (2020)

12. C. Cheng, Y. Jiang, X. Sun, J. Shen, R. Fan, Tunable negative permittivity behavior and electromagnetic shielding performance of silver/silicon nitride metacomposites, Compos. Part A Appl. Sci. Manuf. 130, 105753 (2020)

13. F. Ding, Y. Cui, X. Ge, Y. Jin, S. He, Ultra-broadband microwave metamaterial absorber, Appl. Phys. Lett. 100, 103506 (2012)

14. S. Feng, K. Halterman, Parametrically shielding electromagnetic fields by nonlinear metamaterials, Phys. Rev. Lett. 100, 063901 (2008)

15. N. Limberopoulos, A. Akyurtlu, K. Higginson, A.-G. Kussow, C.D. Merritt, Negative refractive index metamaterials in the visible spectrum based on $\mathrm{MgB}_{2} / \mathrm{SiC}$ composites, Appl. Phys. Lett. 95, 186 (2009)

16. H. Chen, L. Ran, D. Wang, J. Huangfu, Q. Jiang, J.A. Kong, Metamaterial with randomized patterns for negative refraction of electromagnetic waves, Appl. Phys. Lett. 88, 031908 (2006)

17. K. Hur, Y. Francescato, V. Giannini, S.A. Maier, R.G. Hennig, U. Wiesner, Three-dimensionally isotropic negative refractive index materials from block copolymer self-assembled chiral gyroid networks, Angew. Chem. Int. Ed. 50, 11985 (2011) 
18. G.V. Eleftheriades, A.K. Iyer, P.C. Kremer, Planar negative refractive index media using periodically LC loaded transmission lines, IEEE Trans. Microw. Theory 50, 2702 (2002)

19. S. Chui, L. Hu, Theoretical investigation on the possibility of preparing left-handed materials in metallic magnetic granular composites, Phys. Rev. B 65, 144407 (2002)

20. X. Ao, S. He, Negative refraction of left-handed behavior in porous alumina with infiltrated silver at an optical wavelength, Appl. Phys. Lett. 87, 101112 (2005)

21. Z. Shi, R. Fan, Z. Zhang, H. Gong, J. Ouyang, Y. Bai, X. Zhang, L. Yin, Experimental and theoretical investigation on the high frequency dielectric properties of $\mathrm{Ag} / \mathrm{Al}_{2} \mathrm{O}_{3}$ composites, Appl. Phys. Lett. 99, 032903 (2011)

22. Z.c. Shi, R.h. Fan, Z.d. Zhang, L. Qian, M. Gao, M. Zhang, L.t. Zheng, X.h. Zhang, L.w. Yin, Random composites of nickel networks supported by porous alumina toward double negative materials, Adv. Mater. 24, 2349 (2012)

23. T. Tsutaoka, T. Kasagi, S. Yamamoto, K. Hatakeyama, Low frequency plasmonic state and negative permittivity spectra of coagulated $\mathrm{Cu}$ granular composite materials in the percolation threshold, Appl. Phys. Lett. 102, 181904 (2013)

24. T. Tsutaoka, T. Kasagi, S. Yamamoto, K. Hatakeyama, Double negative electromagnetic property of granular composite materials in the microwave range, J. Magn. Magn. Mater. 383, 139 (2015)

25. T. Tsutaoka, K. Fukuyama, H. Kinoshita, T. Kasagi, S. Yamamoto, K. Hatakeyama, Negative permittivity and permeability spectra of $\mathrm{Cu} /$ yttrium iron garnet hybrid granular composite materials in the microwave frequency range, Appl. Phys. Lett. 103, 261906 (2013)
26. H. Massango, T. Tsutaoka, T. Kasagi, S. Yamamoto, K. Hatakeyama, Coexistence of gyromagnetic resonance and low frequency plasmonic state in the submicron Ni granular composite materials, J. Appl. Phys. 121, 103902 (2017)

27. B. Zhao, C.B. Park, Tunable electromagnetic shielding properties of conductive poly (vinylidene fluoride)/ $\mathrm{Ni}$ chain composite films with negative permittivity, J. Mater. Chem. C 5, 6954 (2017)

28. K. Sun, P. Xie, Z. Wang, T. Su, Q. Shao, J. Ryu, X. Zhang, J. Guo, A. Shankar, J. Li, Flexible polydimethylsiloxane/multiwalled carbon nanotubes membranous metacomposites with negative permittivity, Polymer 125, 50 (2017)

29. Z. Wang, K. Sun, H. Wu, P. Xie, Z. Wang, X. Li, R. Fan, Compressible sliver nanowires/polyurethane sponge metacomposites with weakly negative permittivity controlled by elastic deformation, J. Mater. Sci. 55, 15481 (2020)

30. K. Sun, J. Qin, Z. Wang, Y. An, X. Li, B. Dong, X. Wu, Z. Guo, R. Fan, Polyvinyl alcohol/carbon fibers composites with tunable negative permittivity behavior, Surf. Interfaces 21, 100735 (2020)

31. D. Zhang, P. Wang, R.-i. Murakami, X. Song, Effect of an interface charge density wave on surface plasmon resonance in $\mathrm{ZnO} / \mathrm{Ag} / \mathrm{ZnO}$ thin films, Appl. Phys. Lett. 96, 233114 (2010)

32. H. Gu, J. Guo, M.A. Khan, D.P. Young, T. Shen, S. Wei, Z. Guo, Magnetoresistive polyaniline-silicon carbide metacomposites: plasma frequency determination and high magnetic field sensitivity, Phys. Chem. Chem. Phys. 18, 19536 (2016)

33. X. Yao, X. Kou, J. Qiu, Multi-walled carbon nanotubes/ polyaniline composites with negative permittivity and negative permeability, Carbon 107, 261 (2016)

Cite this article as: Yan An, Jinyuan Qin, Kai Sun, Jiahong Tian, Zhongyang Wang, Yaman Zhao, Xiaofeng Li, Runhua Fan, Carbon fiber skeleton/silver nanowires composites with tunable negative permittivity behavior, EPJ Appl. Metamat. 8, 1 (2021) 